\title{
OP05
}

\section{OBSERVER CMF BASED VISUAL APPEARANCE COMPENSATION FOR NOVEL LIGHT SOURCE PROJECTION SYSTEM}

\author{
Chris Yi-Ho Bai et al.
}

DOI 10.25039/x47.2020.OP05

\section{Paper accepted for the $5^{\text {th }}$ CIE Symposium on Colour and Visual Appearance}

The paper was selected by the International Scientific Committee (ISC) for presentation at the 5th CIE Symposium on Colour and Visual Appearance, Hong Kong, CN, April 21-22, 2020, which, due to the corona pandemic, could not take place. The paper has not been peer-reviewed by CIE.

\section{(C) CIE 2020}

All rights reserved. Unless otherwise specified, no part of this publication may be reproduced or utilized in any form or by any means, electronic or mechanical, including photocopying and microfilm, without permission in writing from CIE Central Bureau at the address below. Any mention of organizations or products does not imply endorsement by the CIE.

This paper is made available open access for individual use. However, in all other cases all rights are reserved unless explicit permission is sought from and given by the CIE.

CIE Central Bureau

Babenbergerstrasse 9

A-1010 Vienna

Austria

Tel.: +4317143187

e-mail: ciecb@cie.co.at

www.cie.co.at 


\title{
OP05 \\ OBSERVER CMF BASED VISUAL APPEARANCE COMPENSATION FOR NOVEL LIGHT SOURCE PROJECTION SYSTEM
}

\author{
Bai, C.Y.H. ${ }^{1,2}$, Tseng, C.F. ${ }^{1}$, Lu, T.Y. ${ }^{1,2}$, Ou, L.C. ${ }^{1}$ \\ ${ }^{1}$ Graduate Institute of Applied Science and Technology, National Taiwan University of Science and \\ Technology, Taipei, CHINESE TAIPEI, \\ ${ }^{2}$ Color Technology Lab, BenQ Corporation, Taipei, CHINESE TAIPEI \\ D10022503@mail.ntust.edu.tw
}

\begin{abstract}
As display technology expands the color gamut, the potential of experiencing observer metamerism is more likely with narrow bandwidth stimuli displays. Hence, it is desired to investigate whether laser-based projector would introduce observer metamerism compared to halogen-based projector. In addition, it is also desired to develop a quick method to determine an observer's color matching function category.

In this study, a two-part experiment was designed and conducted. Experiment 1 was based on color patches and Experiment 2 was based on test images. The quick method was developed in Experiment 1. Comparison to CIE Standard Observer was also conducted, and the results had suggested the CIE Standard Observer was not suitable for laser-based projection system.

In Experiment 2, a prediction was made to determine the image category. With the quick method, it is much easier to achieve the realization of personalized color management system, hence, reducing the issue of observer metamerism.
\end{abstract}

Keywords: Observer Metamerism, Observer Category, Projector

\section{Motivation and Introduction}

As technology evolves, novel light source has been introduced in projection systems to save energy and to expand colour gamut. Novel light source can be LED or Laser based. In order to achieve the two objectives, the light source has to be very efficient in matching human luminous efficiency function $V(\lambda)$, and also has very narrow bandwidth in $R, G$, and $B$ primaries. However, an unpredictable drawback is the increased degree of observer metamerism (Asano, 2014). The phenomenon is exhibited when two stimuli with very different spectral power distribution can generate the same cone response (Sarkar, 2010), leading to the same tristimulus values. This is called a metameric match. However, the match is often valid only with one observer and exhibits as a mismatch to another observer under the same condition. Since the second observer has a set a different colour matching functions (CMFs) than the first observer, hence, the metameric match fails, and this is commonly referred as observer metamerism (Chen, 2019).

Observer metamerism may cause an issue in real world applications such as soft proofing and colour grading scenarios. In Alfvin and Fairchild (1997), and Rich and Jalijali's (1995) work, both studies has shown large observer variability which exhibited a serious issue for soft proofing scenario. Asano et al. (2014) utilized colour images in the colour matching experiment on a CCFL backlight LCD monitor and a laser projector for colour grading purpose. The result has also shown that inter-observer variability is larger than intra-observer variability, which indicates observer metamerism can cause a serious issue in colour grading with laser projector.

Since observer metamerism is affecting some real-world applications, it is necessary to look at the solution to this issue. One solution to this issue is to develop a display device which can allow customized CMFs to replace the CIE Standard Observer. The key is how to obtain the personalized CMFs for individual observers. A practical approach to estimate one's CMFs is through predetermined observer CMFs or observer classification since it is not trivial to determine one's CMFs. Sarkar et al. (2010) had developed a method for establishing 8 
colorimetric observer categories with two-step algorithm using two spectrally distinctive displays, one CRT monitor and one LED backlight monitor. Colour patches were predicted by different observer categories and displayed on the two displays, and the participants were asked to choose the best matching pair through the experiment procedure. Several base colours were repeated for each participant, and the final observer category was determined by an empirical ranking system to achieve good colour matching among most base colour pairs.

Asano (2015) improved on Sarkar's method, but derived 10 observer categories for his work. A two-step method was utilized in his work, too. A set of 10000 individual observer CMFs was generated from Monte Carlo simulation, and then a cluster analysis was applied to determine the 10 observer categories (Chen, 2019).

In this study, it is desired to match the colour appearance of the laser-based projection system to the colour appearance of halogen lamp-based projection system. Therefore, it is desired to devise a method to quickly categorize observer's CMFs based on the colours which observer believes they are matched under the two different projection systems. Based on the category, it is desired that the same method can be applied to predict the observer category for images.

\section{Experiment}

The experiment procedure will be discussed in this section.

\subsection{Apparatus Overview}

The apparatus used in the experiment is listed and the specification is described in the following:

a. Halogen Lamp Projection System:

- Model: BenQ LX810STD

b. Blue Laser Projection System:

- Model: BenQ MX600

c. Projection Screens:

- Model: Elite Screens

- 100" 16:9 aspect ratio (Height * Width: $185 \mathrm{~cm}{ }^{*} 104 \mathrm{~cm}$ )

- Gain: 1:1

- Viewing Angle: $160^{\circ}$

\subsection{Quick Method to Determine Observer Colour Matching Functions}

From previous studies, it is desired to determine observer's colour matching function for each person. However, to determine the exact colour matching function often takes enormous amount of time and energy. Hence, it is much more desirable to devise a method to quickly categorize observer's colour matching functions.

The logic behind devising the quick method is described in the following:

The deviation in each individual observer will cause perceived colour difference among different observers even the measured values are the same. The phenomenon is even more sever on modern narrow bandwidth display devices. The idea is to pre-determine a set of colour matching function categories, and using a selection method to determine an observer's category on the novel display. The selection method involves two types of display, one is the narrow bandwidth display, and another is the broad bandwidth display. Observer population simulation, colour patch selection, pre-determined set of colour matching function categories, and method of category determination are also a part of the selection method, and these will be discussed thoroughly in the following sections.

\subsubsection{Observer Population Simulation}

In order to obtain the variation of observer CMFs, it is necessary to access the CMFs of a large number of populations. For simplicity of this study, the Monte Carlo Simulation of Observer Colour Matching Functions from RIT was utilized. A 10000 observers were generated using the generator with age between 20 to 30 years old, which fits with the participants age group in both experiment 1 and 2 . The field size was set at 10 degrees for colour patch matching. The observer variation in colour matching functions is illustrated in Figure 2, where red lines 
represents $\mathrm{X}$ bar functions, green lines represents $\mathrm{Y}$ bar functions, and blue lines represents $\mathrm{Z}$ bar functions.

\subsubsection{Colour Patch Selection}

Colour patches are selected to determine observer's CMFs. However, it is not possible to randomly select colour patches to fulfil the purpose. Therefore, rules had been established based on the population simulated to obtain the variation of observer CMFs to select colour patches for determine a particular observer's CMFs:

1. Larger variation between observers in CMFs,

2. Wavelengths where $r, g, b$ curves intersect, and

3. Two points near the $380 \mathrm{~nm}$ and $780 \mathrm{~nm}$ where the devices can reproduce the colours.

The selection process is first based on observer variation in CMFs graph obtained from Monte Carlo Simulation, and then verified on characterization model to determine if the device could reproduce the colour accurately. The final wavelengths selected are: $418 \mathrm{~nm}, 463 \mathrm{~nm}, 472 \mathrm{~nm}$, $495 \mathrm{~nm}, 510 \mathrm{~nm}, 545 \mathrm{~nm}, 558 \mathrm{~nm}, 565 \mathrm{~nm}, 571 \mathrm{~nm}, 585 \mathrm{~nm}, 600 \mathrm{~nm}, 606 \mathrm{~nm}, 630 \mathrm{~nm}$, and $660 \mathrm{~nm}$. Total of 14 colour patches are selected. The selection is also plotted on Figure 2.

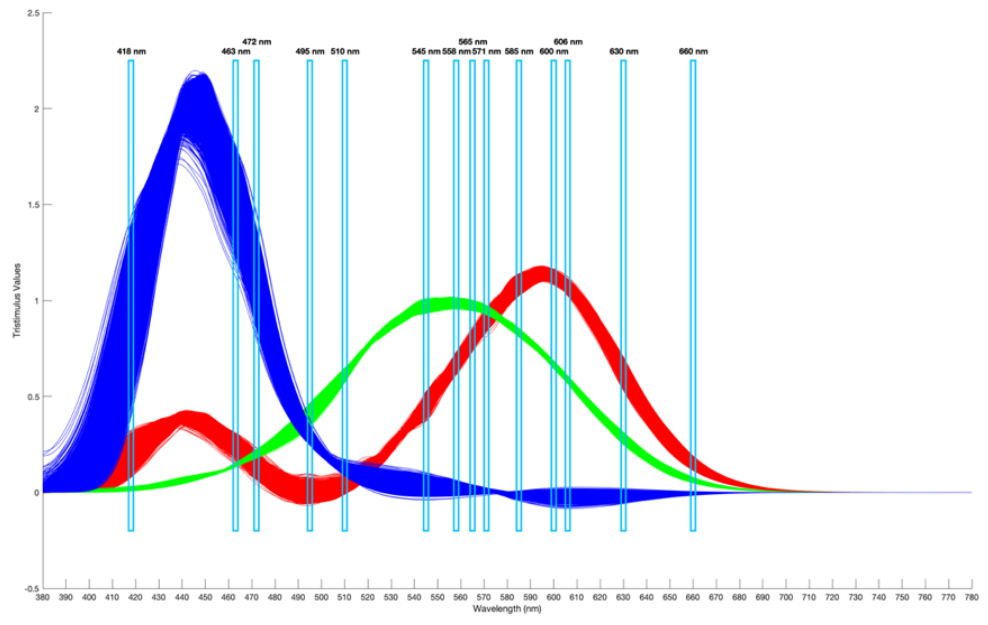

Figure 2 - Final Wavelength Selection based on Monte Carlo Simulation Result

\subsubsection{Pre-Determined Colour Matching Function Categories}

The pre-determined colour matching function categories are constructed based on the wavelength selected and Monte Carlo Simulation. Seven categories were constructed from the data set with one category consists of the same data as CIE Standard Observer Colour Matching Functions. The other six categories were determined by evenly divided the variation among the observers' colour matching functions from Monte Carlo Simulation, and all seven categories of pre-determined colour matching function categories are illustrated in Figure 3.

\subsubsection{Method of Category Determination}

The method of category determination used in the experiment is utilizing 'mode' to determine the final category of one observer. The reason behind is it is logical to choose the 'most chosen' category of a particular observer to represent his or her category since the category was chosen when the colour was a match according to the observer. 'Mean' or 'average' category does not represent a meaningful interpretation in this context. For example, if an observer selects the following categories after the experiment: "category 2, category 1 , category 2, category 2 , category 2 , category 3 , category 7 , category 5 , category 2 , category 4 , category 2 , category 2 , category 1 , category 2 ", the result would be "category 2 " since the most selected category is "category 2". 


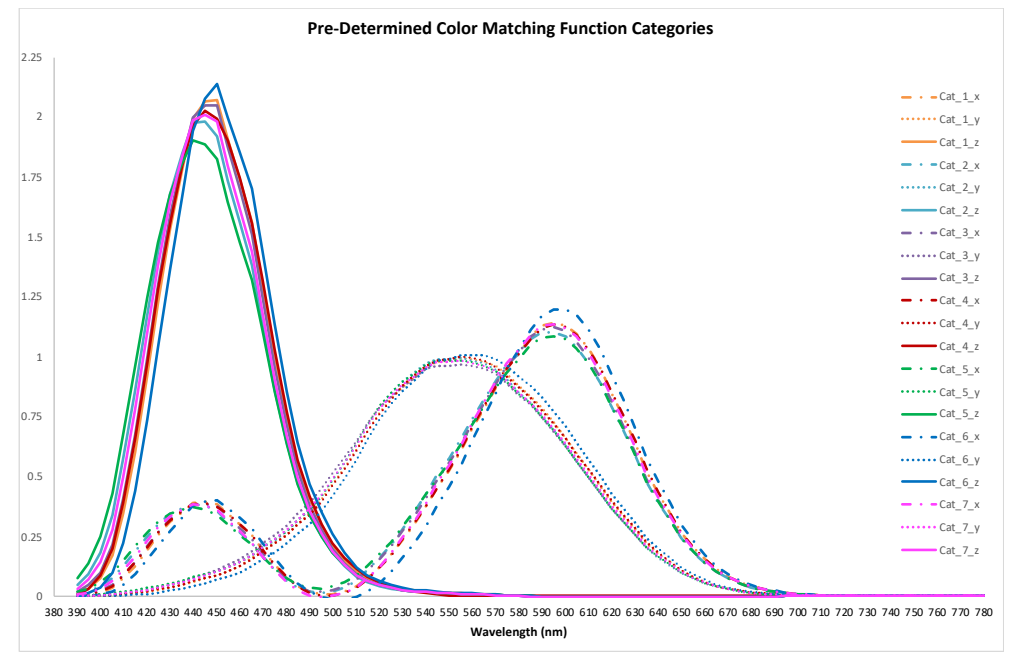

Figure 3 - Pre-Determined Seven Colour Matching Function Categories in xyz Curves

\subsection{Experiment 1: Using the Quick Method to Determine Observer Colour Matching Function}

A MatLAB program was developed to conduct the experiment using the quick method to determine observer's CMFs. Each session takes about 30 minutes to complete the required task. The experiment setup is illustrated in Figure 4. Please note the distance between the projection screens and the two projection systems are not the same since the focal lengths are not the same for the two projection systems. In order to display the same image size, the distance has to be different. The experiment was conducted in a dark environment, with blue laser projection system projects on the left screen, and halogen lamp projection system projects on the right screen. The halogen lamp projection system servers as the 'reference' display, hence, the colour patches projected are the 'reference patches'. There are 14 reference patches for each observer, and for each reference patch, there are 7 colour patches projected by the blue laser projection system for the observer to choose from. Observer can only choose one colour patch from the 7 colour patches for each reference patch, and has to choose one. All reference patches were shown randomly, and repeated for 3 times. As a result, each observer needs to conduct 42 colour matches. The flow chart of Experiment 1 is illustrated in Figure 5.

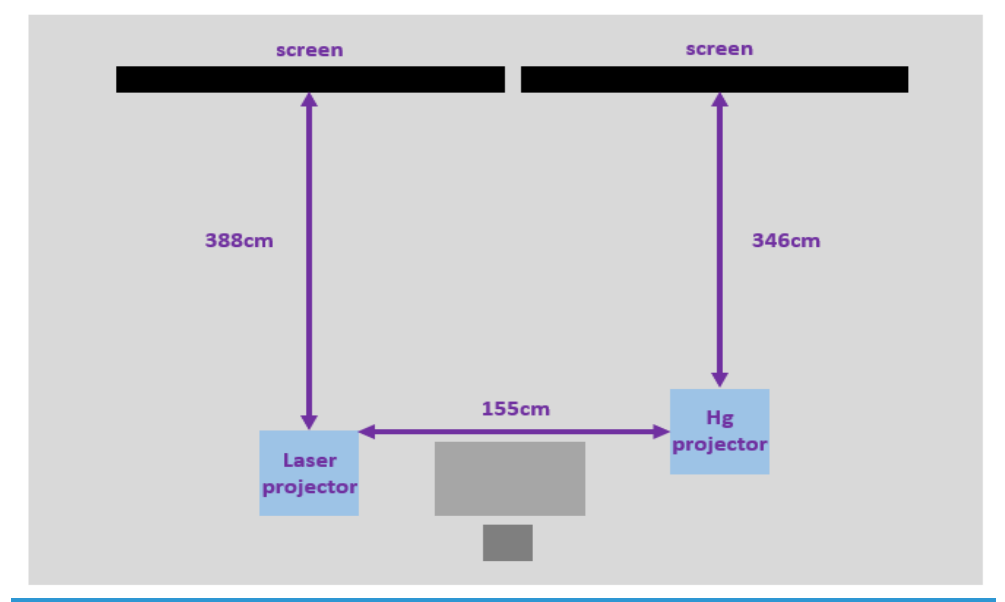

Figure 4 - Illustration of Experiment Setup

On the blue laser projection system screen, there are three buttons to control the flow of the experiment, namely 'Previous', 'Next', and 'Match'. Observer can click on the corresponding button to select the colour patch using 'Previous' or 'Next', or determine if the selected colour patch is a match to the reference patch. If the observer click on 'Match', then the software program will record the colour patch which is a match and display the next reference patch. Between each reference patch, a gray patch will display for 5 second on both projection system 
to avoid visual residual effect. The program will continue to display all 42 reference patches and ask for observer's input.

After observer finish all 42 judgement, the program will determine observer's category. However, observer will not know his or her own category since the extra information may interfere with the next experiment.

\subsection{Experiment 2: Refining Pre-Determined Categories and Applying Observer Colour Matching Function to Images}

There are two goals to be fulfilled in Experiment 2. The first goal is to verify the individual observer colour matching function category can also be applied to images, and it also performs better than the Standard Observer CMFs. The second goal is to utilize the quick method devised in Experiment 1 and to verify the observer category determined by the quick method could correlate to the image category that the observer chosen. The flow chart of Experiment 2 is illustrated in Figure 6. A MatLAB program was developed to conduct the experiment. A set of images was pre-selected and rendered for different observer colour matching function categories. The set of images used in the experiment consists of 8 images and is exhibited in Figure 7.

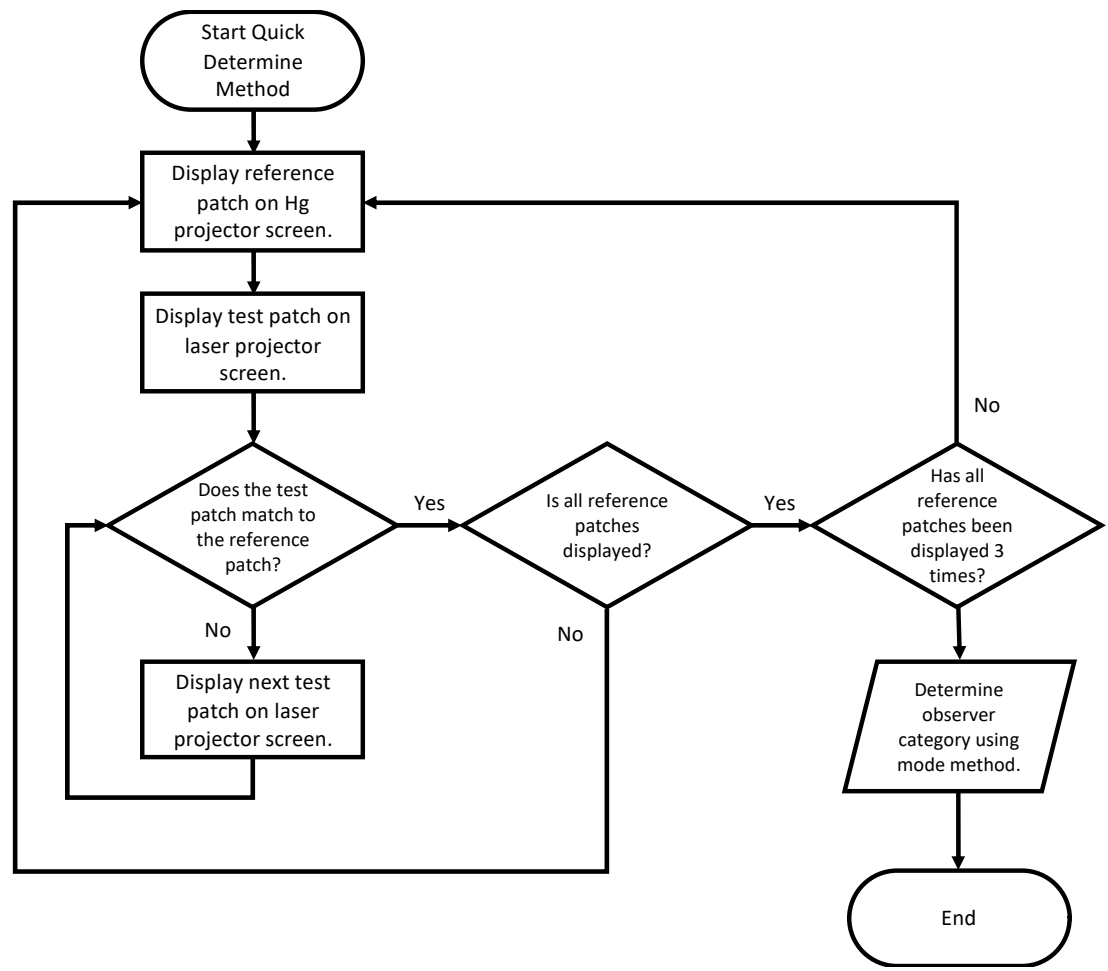

Figure 5 - Flow Chart of the Quick Method to Determine Observer Colour Matching Function

The selection of the 8 images consists of natural colours such as sky, grass, flowers, trees and sunset, skin tone colours, familiar objects, such as cakes, wine bottles, fabrics, music instrument, boats, yarns, crayons, and standard colour patches and neutral colours.

From Experiment 1, it could be concluded that the result could be further grouped into fewer categories. This trend could help further simplifying the experiment. By grouping into fewer number of new categories, observers could be more focused and judging less comparison. Observers were asked to conduct a quick method of colour patch determination first (without repetition), and then followed by the image experiment part. The same experiment setup, environment and procedure as in Experiment 1 were taken in Experiment 2. The only differences were using images and fewer categories in the image experiment part. Each image was repeated for 3 times, hence, a total of 24 judgement was made for each observer. After the observer finish all 24 judgement, the program will also determine the observer's category for 
the image judgement. A comparison with the colour patch category will also be conducted in order to validate the usefulness of the quick method devised in Experiment 1.

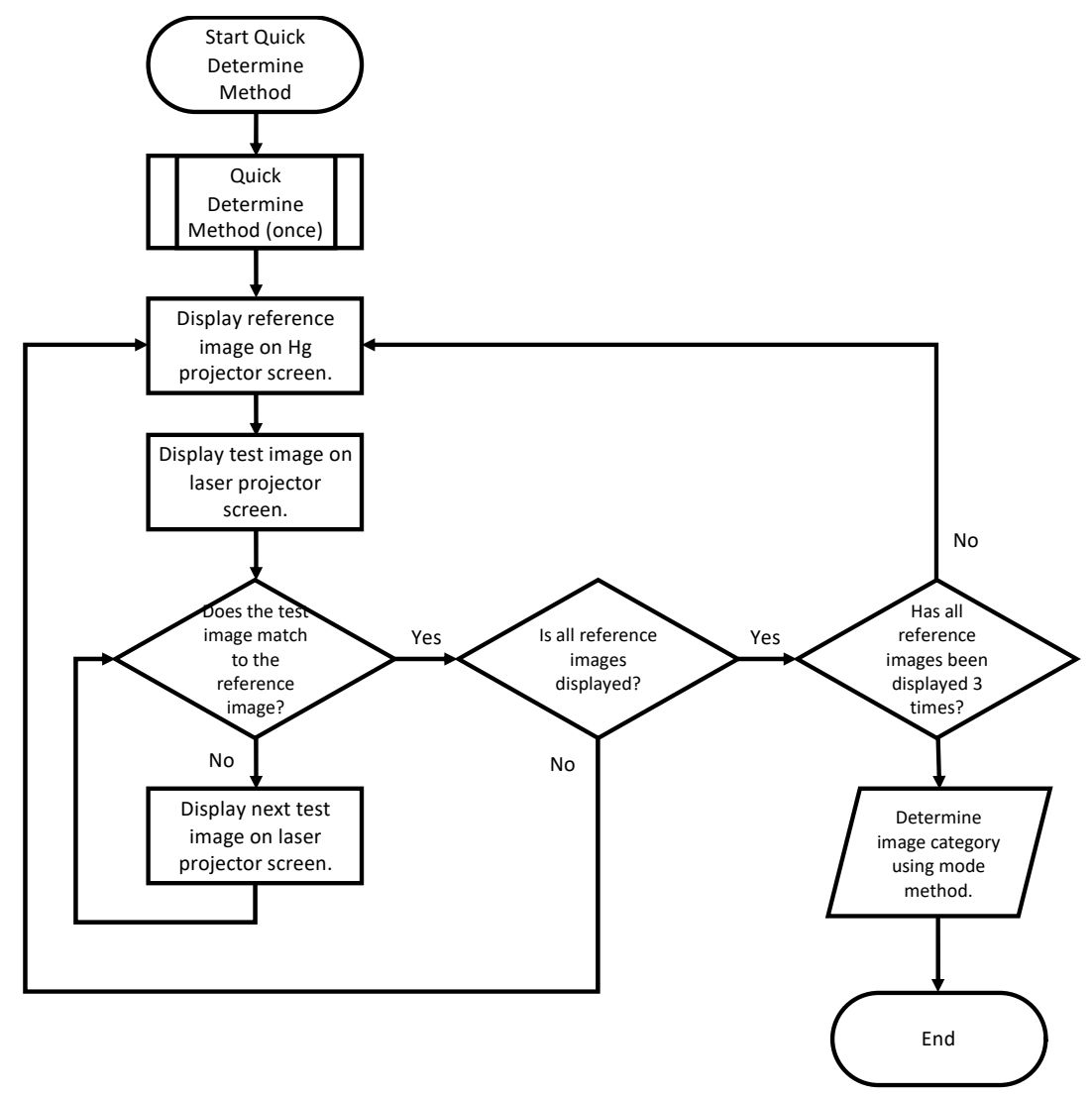

Figure 6 - Flow Chart of Determine Image Category in Experiment 2

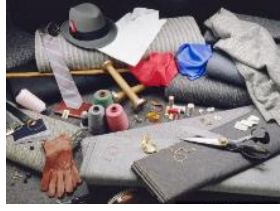

Test Image 1

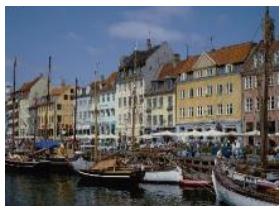

Test Image 5

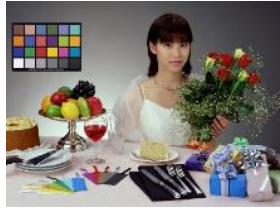

Test Image 2

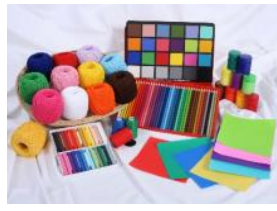

Test Image 6

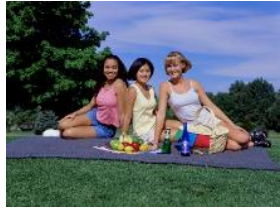

Test Image 3

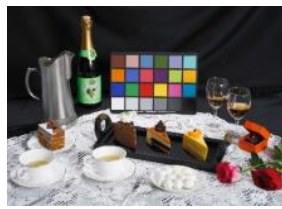

Test Image 7

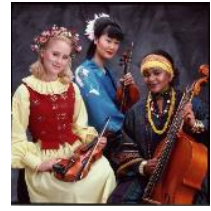

Test Image 4

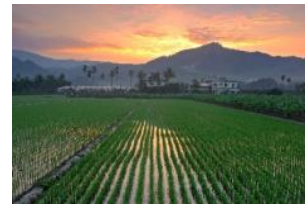

Test Image 8

Figure 7 - Eight Test Images Used in the Experiment Consisting of Natural Colours, Skin Tones, Familiar Colours and Standard Colour Patches and Neutral Colours

In summary, Experiment 1 investigates the feasibility of the quick method of determining observer colour matching function category using halogen and laser projection systems. In Experiment 2, it will first utilize the quick method to determine the colour matching function category, then conduct the image experiment to determine the image category. It is expected that the quick method could predict the outcome of the image category, and this hypothesis would be verified in Experiment 2. Also, the outcome of both experiments will determine if the CIE Standard Observer is suitable for this group of observer or not.

\section{Results and Discussions}

In this section, the results and the findings of the experiment will be discussed. 


\subsection{Results of Experiment 1}

Fifty participants were involved in Experiment 1 with 28 male and 22 female university students (age between 20 to 30 ). When combining all 14 colour patches results together, the response could be plotted in a histogram shown in Figure 8. In Figure 8, the number of categories is plotted against of the responses, and can be concluded that category 5 has the most responses, whereas category 2 has the least responses. Category 4 represents the CIE $196410^{\circ}$ Standard Observer; however, it does not have the most responses. This leads to the conclusion that the CIE $196410^{\circ}$ Standard Observer may not be the optimum colour matching function for this group of observers.

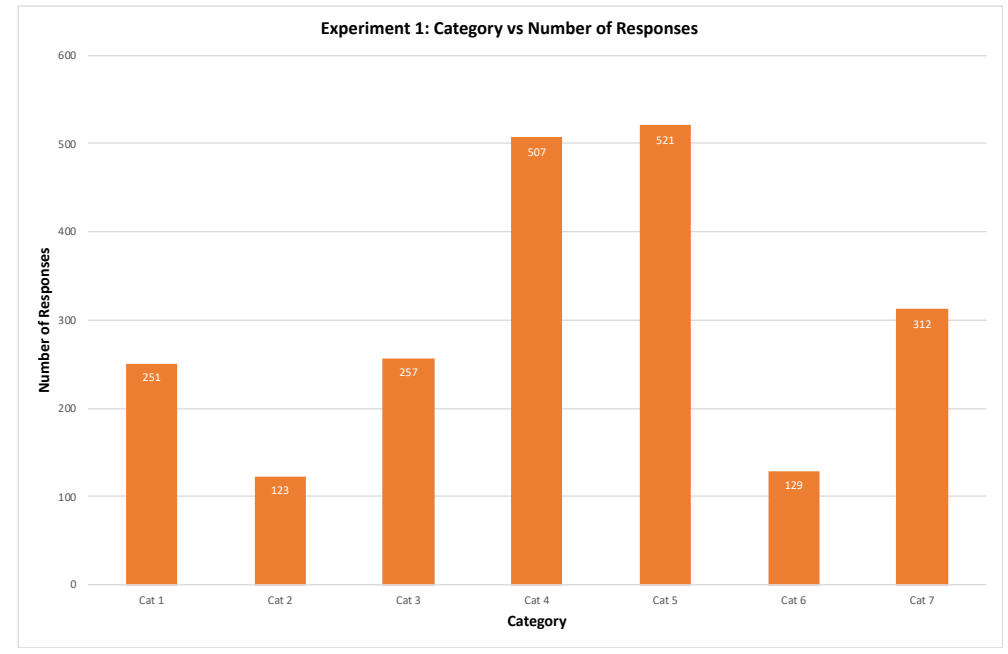

Figure 8 - Category versus Number of Responses for Experiment 1 Result

When all fifty observers' responses are plotted against in terms of $X, Y$ and $Z$ curves, as shown in Figure 9 (a), (b) and (c), respectively, $X$ and $Z$ curves exhibit a clear pattern which can divide all the responses into three groups. Hence, based on the pattern, the $X, Y$, and $Z$ curves could be divided into three categories and shown in Figure 10 (a), (b) and (c) respectively. The median number was taken to obtain the final $X, Y$ and $Z$ curves.

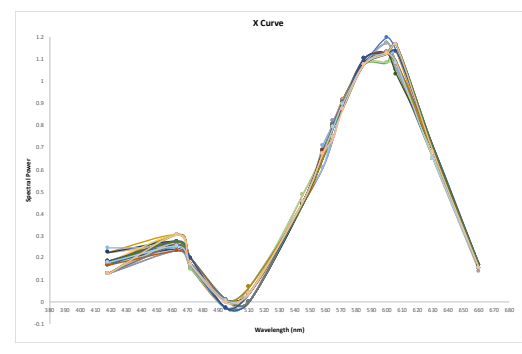

(a)

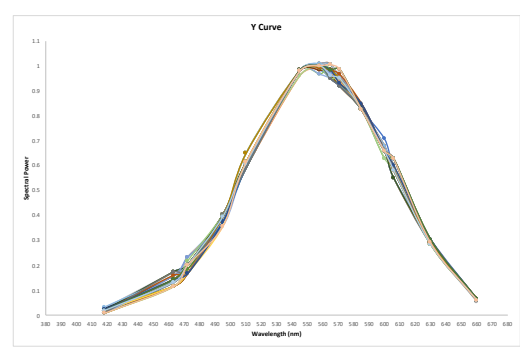

(b)

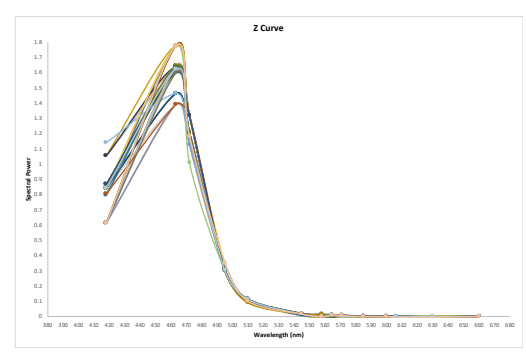

(c)

Figure 9 - (a) All 50 Observers' Responses Plotted in Terms of X Curves. (b) All 50 Observers' Responses Plotted in Terms of Y Curves. (c) All 50 Observers' Responses Plotted in Terms of $Z$ Curves.

\subsection{Results of Experiment 2}

Thirty participants were involved in Experiment 2 with 18 male and 12 female university students (age between 20 to 30). After the new four observer categories were established (three new observer categories plus the CIE $19312^{\circ}$ Standard Observer), all the test images were transformed to the new observer categories accordingly. A series of psychophysical experiment were conducted to determine the best matched colour matching function for this group of observers. 


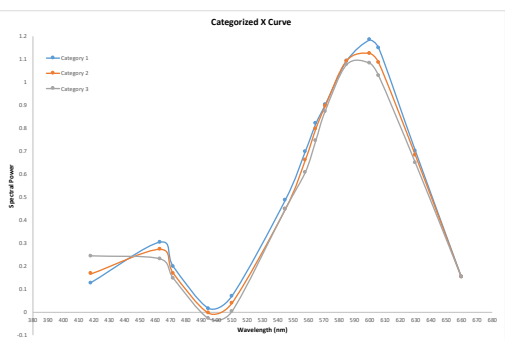

(a)

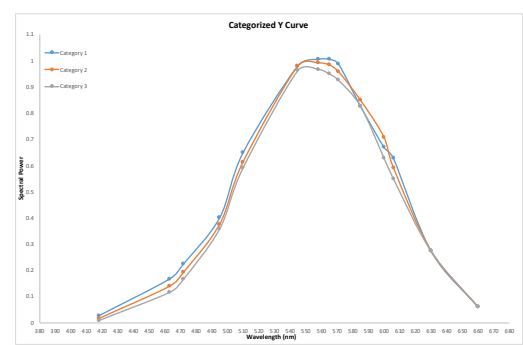

(b)

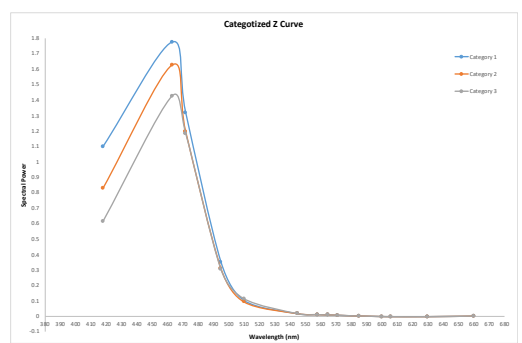

(c)

Figure 10 - (a) Categorized X Curves for All 50 Observers' Responses. (b) Categorized Y Curves for All 50 Observers' Responses. (c) Categorized Z Curves for All 50 Observers' Responses.

\subsubsection{Image Preparation}

Eight test images were selected for Experiment 2, and the images are exhibited in Figure 7. Test images were carefully selected to enclosed as many hues and scenarios as possible, including grayscale, skin tone, natural scene, familiar and colourful objects. All the test images were transformed by the image pipeline defined in Figure 11.

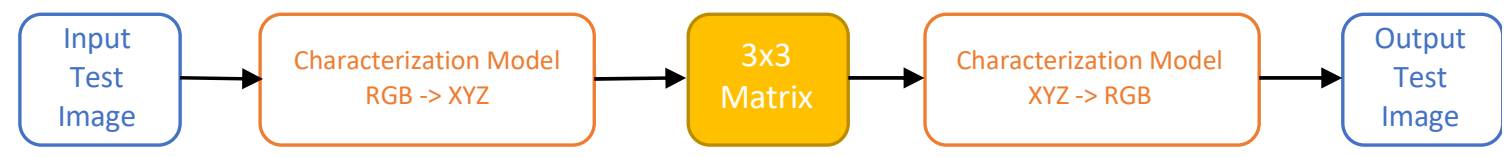

Figure 11 - Image Pipeline for Transforming Test Images from Standard Observer to New Observer Categories

\subsubsection{Data Analysis for Experiment 2 Result}

The first goal of Experiment 2 was to verify the individual observer colour matching function category can also be applied to images, and it also performs better than the CIE $19312^{\circ}$ Standard Observer colour matching function. The result of all thirty observers' responses is shown in Figure 12. From Figure 12, it is obvious to determine that Category 2 was the most representative category for this group of observers. Category 4 represents the CIE $19312^{\circ}$ Standard Observer; hence, it can be concluded that the new observer category is more preferred than the CIE $19312^{\circ}$ Standard Observer on the blue laser projection system.

The second goal of Experiment 2 was to utilize the quick method devised in Experiment 1 and to verify the observer category determined by the quick method could correlate to the image category that the observer chosen. The relationship between the new image category used in Experiment 2 and original observer category used in Experiment 1 is described in Table 2. Based on the relationship provided in Table 2, the outcome of the quick method from Experiment 1 had been changed to the new image categories. The results of Experiment 2 are summarized in Table 3. The response from the quick method after converted to the new image categories, the image categories determined from Experiment 2, and whether the predicted category matched to the actual category is indicated in Table 3. There were total of 30 observers participated in Experiment 2, and 18 responses were 'Yes', and 12 responses were 'No'. The prediction successful rate is $60.0 \%$. The performance of the quick method is not bad given the complex context it needs to handle. But there are certain places can be improved in the future version: 


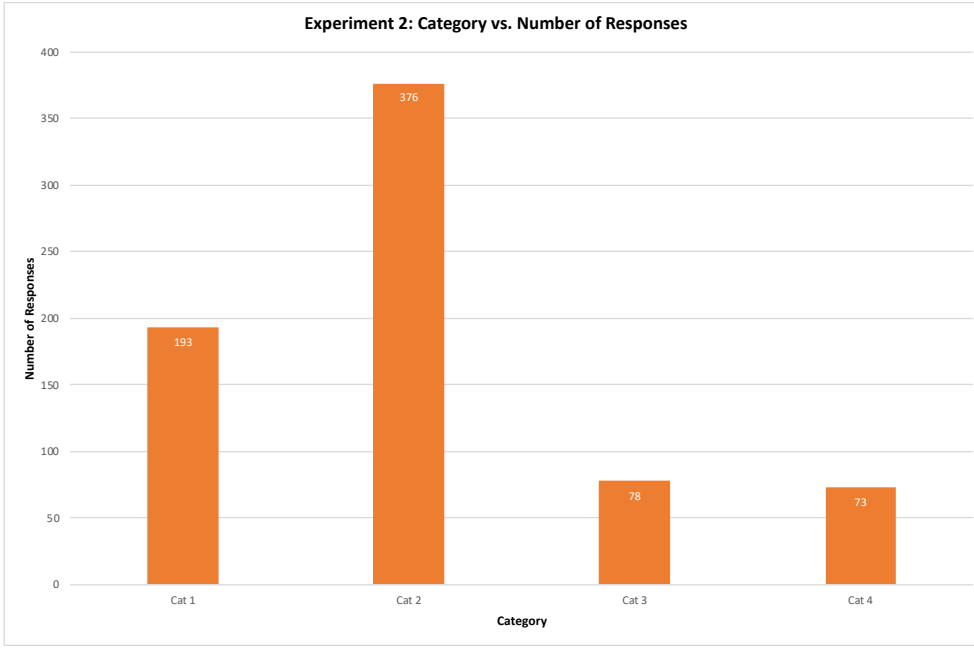

Figure 12 - Category versus Number of Responses for Experiment 2 Result

1) For the quick method to predict the image category, use field size of $2^{\circ}$ to conduct the matching rather than $10^{\circ}$.

2) Use a more accurate characterization model for the projection system so that the RGB values interpolated could correspond to the exact wavelength required. A $13 \times 13 \times 13$ 3D LUT is recommend for this purpose.

3) Eliminate reference colour patches with high 'inconsistent' response rate. Inconsistent response may indicate observers could not find the 'correct' matching test colour patch.

Table 2 - Relationship between the New Image Category and Original Observer Category Used in Experiment 1

\begin{tabular}{|l|l|}
\hline New Image Category & Original Category \\
\hline Category 1 & Category $1 \& 3$ \\
\hline Category 2 & Category $2,5 \& 7$ \\
\hline Category 3 & Category 6 \\
\hline Category 4 & $\begin{array}{l}\text { CIE 1931 Standard } \\
\text { Observer }\end{array}$ \\
\hline
\end{tabular}

In summary, two goals set for Experiment 2 were met. The new image category 2 was found to be better suited for the group of observers participated in the experiment than using the CIE $19312^{\circ}$ Standard Observer. And it was found to be possible to predict the image category from the quick method devised from the colour patch. Although the success rate is $60.0 \%$, with a few improvements in the future, it could be a viable method to determine observer's individual colour matching category.

Table 3 - Experiment 2 Results Using the Quick Method to Predict Observer's Image Category

\begin{tabular}{|l|c|c|c|c|c|c|c|c|c|c|c|c|c|c|c|}
\hline Obs & 1 & 2 & 3 & 4 & 5 & 6 & 7 & 8 & 9 & 10 & 11 & 12 & 13 & 14 & 15 \\
\hline Exp 1 & 2 & 1 & 1 & 2 & 2 & 1 & 2 & 1 & 2 & 1 & 2 & 2 & 1 & 1 & 2 \\
\hline Exp 2 & 2 & 1 & 1 & 2 & 2 & 2 & 2 & 2 & 2 & 2 & 2 & 2 & 1 & 2 & 2 \\
\hline Match & $\mathrm{Y}$ & $\mathrm{Y}$ & $\mathrm{Y}$ & $\mathrm{Y}$ & $\mathrm{Y}$ & $\mathrm{N}$ & $\mathrm{Y}$ & $\mathrm{N}$ & $\mathrm{Y}$ & $\mathrm{N}$ & $\mathrm{Y}$ & $\mathrm{Y}$ & $\mathrm{Y}$ & $\mathrm{N}$ & $\mathrm{Y}$ \\
\hline Obs & 16 & 17 & 18 & 19 & 20 & 21 & 22 & 23 & 24 & 25 & 26 & 27 & 28 & 29 & 30 \\
\hline Exp 1 & 2 & 2 & 1 & 4 & 2 & 2 & 2 & 4 & 4 & 1 & 2 & 1 & 1 & 1 & 1 \\
\hline Exp 2 & 1 & 2 & 1 & 2 & 1 & 2 & 2 & 2 & 2 & 2 & 2 & 1 & 1 & 2 & 2 \\
\hline Match & $\mathrm{N}$ & $\mathrm{Y}$ & $\mathrm{Y}$ & $\mathrm{N}$ & $\mathrm{N}$ & $\mathrm{Y}$ & $\mathrm{Y}$ & $\mathrm{N}$ & $\mathrm{N}$ & $\mathrm{N}$ & $\mathrm{Y}$ & $\mathrm{Y}$ & $\mathrm{Y}$ & $\mathrm{N}$ & $\mathrm{N}$ \\
\hline
\end{tabular}




\section{Conclusion}

While display technology advances, the enlargement in colour gamut is a particular item needs to be drawn attention to. Large colour gamut could produce to more vivid colours, but also incorporate narrow bandwidth primaries and could lead to higher potential of observer metamerism. Laser projection system is one particular device that suffering from this phenomenon severely. A solution to this is to incorporate individual observer's CMFs in the projection system. Hence, this study focused on whether a quick method could be devised to predict an observer's colour matching function category.

Two experiments were designed and conducted in this study; Experiment 1 was based on 14 reference colour patches, and Experiment 2 was based on 8 test images. After both experiments were conducted, it was found that the current CIE Standard Observer is not suitable for the laser-based projection system. A different observer category was chosen in both experiments for this group of observers. Hence, this concludes that observer metamerism was introduced in laser projection system more severely than halogen-based projection system.

There was a $60.0 \%$ success rate to predict the image category from the quick method using colour patches in Experiment 2. The success rate could be improved if the field size of the colour patches were changed from $10^{\circ}$ to $2^{\circ}$, a better characterization model was introduced, and the colour patches with high inconsistency were eliminated. But it is still encouraging that a few simple colour matching trials could determine one's colour matching function category. This is a one missing piece from developing a personalized colour management system in order to have the optimal viewing experience and accuracy.

\section{References}

Alfvin R.L., Fairchild M.D., (1997). Observer variability in metameric color matches using color reproduction media. Color Research and Application. 22(3), 174-188.

Asano, Y., Fairchild, M., Blond'e, L., Morvan, P., (2014). Observer variability in color image matching on a LCD monitor and a laser projector. 22nd Color and Imaging Conference and 2nd Congress of the International Academy of Digital Pathology. November 14, 2014. Boston, Massachusetts, USA. Springfield: Society of Imaging Science and Technology, pp. 1-6.

Asano Y., (2015). Individual Colorimetric Observers for Personalized Color Imaging. PhD. thesis, Rochester Institute of Technology.

Chen Q., Feng L., Li Y., Cai S., (2019). Reviews on Observer Metamerism and Individual Color Vision Variability. In: P. Zhao, Y. Ouyang, M. Xu, L. Yang, Y. Ren, eds. Advances in Graphic Communication, Printing and Packaging, Proceedings of $20189^{\text {th }}$ China Academic Conference on Printing and Packaging. Berlin: Springer. pp. 23-30.

Rich D.C., Jalijali J., (1995). Effects of observer metamerism in the determination of human color-matching functions. Color Research and Application. 20(1), 29-35.

Sarkar A., Blond'e L., Callet P.L., Autrussear F., Morvan P., Stauder J., (2010). A color matching experiment using two displpays: design considerations and pilot test results. Fifth European Conference on Color in Graphics, Imaging and Vision, CGIV. June 2010, Joensuu, Finland. Springfield: Society of Imaging Science and Technology, pp. 414-422. 